\title{
Handgrip strength and associated sociodemographic and lifestyle factors: A systematic review of the adult population
}

\author{
Tiago Rodrigues de Lima, PE, MSc ${ }^{\text {a,*, }}$ \\ Diego Augusto Santos Silva, PE, PhD a, \\ João Antônio Chula de Castro, $\mathrm{PE}, \mathrm{MSc}{ }^{\mathrm{a}}$, \\ Diego Giulliano Destro Christofaro, PE, PhD ${ }^{\mathrm{b}}$
}

\author{
a Federal University of Santa Catarina, Physical Education Department, Florianopolis, SC, Brazil \\ b Paulista State University "Júlio de Mesquita Filho", Faculty of Science and Technology from \\ Presidente Prudente, Presidente Prudente, SP, Brazil
}

Received 27 June 2016; received in revised form 18 August 2016; accepted 30 August 2016

\section{KEYWORDS \\ Adult health; Motor activity; Muscle strength dynamometer; Public health; Review}

\begin{abstract}
Summary Objetives: The aim of this study was to identify studies on handgrip strength (HGS) and associations with sociodemographic variables and lifestyle in adults.

Methods: Searches were performed in Scielo, PubMed, EBSCO, Lilacs, Scopus and Web of Science databases.

Data extraction: Two blinded reviewers independently screened the articles, scored their methodological quality and extracted data.

Quality assessment: The selected studies were analyzed according to the agreement of their findings with the evidence summary.

Results: Overall, 18,038 studies were found and 26 articles were selected. Lower HGS levels were found in older individuals $(n=20)$, females $(n=13)$ and in those not engaged in physical activities $(n=5)$.

Conclusions: Older adults, females, those of lower educational level and not physically active had lower HGS levels. The adoption of standardization in relation to specific cutoff points for HGS classification becomes necessary in order to allow better comparison of results.

(c) 2016 Elsevier Ltd. All rights reserved.
\end{abstract}

\footnotetext{
* Corresponding author. Professor Clementino de Britto, 362 ap 334 Capoeiras, Florianópolis, Santa Catarina 88019-150, Brazil. E-mail address: tiagopersonaltrainer@gmail.com (T.R. de Lima).
} 


\section{Introduction}

Handgrip strength is the sum of the strength of the flexor muscles against the palmar (Mathiowetz et al., 1985). The extensor muscles act in a secondary way in order to assist the intrinsic muscles contribution playing a minor role in the generation of the grip strength (Mathiowetz et al., 1985). Despite being used to evaluate hand function, disease severity, check the effectiveness of a particular treatment or decide on the suitability of a subject to return to work, grip strength measurement also reflects general health and level of physical activity of the individual, being a low-cost tool to predict overall strength (Hansen et al., 2013; Leong et al., 2015; Rantanen et al., 1992).

Several factors are associated with decreased muscle strength, among them, loss of muscle mass and reduction of skeletal muscle fibers that become more compromised with increasing age (Abe et al., 2014). In addition to these factors, the decrease in serum levels of testosterone and adrenal androgens, increased action of inflammatory mediators (IL-1 and IL-6) and factors related to the change in protein synthesis and consequent reduction in $\mathrm{GH}$ and IGF-1 secretion are related to lower muscle strength levels (Montalcini et al., 2013; Schlüssel et al., 2008).

Low handgrip strength levels, besides causing limitations related to the loss of physical function, is associated with increased health recovery time after illness or surgery, malnutrition, type II diabetes, cardiovascular complications and overall mortality (Leong et al., 2015; Montalcini et al., 2013; Schlüssel et al., 2008).

The investigation of sociodemographic and lifestyle factors that are associated with lower handgrip strength levels can provide specific characteristics in relation to population subgroups that should be postponed in relation to the planning of prevention strategies and coping with health diseases, and to identify pre determining risk factors associated with low levels of this valence (Leong et al., 2015).

Research has shown that populations subgroups of adults who have lower handgrip strength levels were composed of females, individuals over 40 years and 50 years for males, people of lower economic level and lower educational level, those not engaged in regular physical activities, smokers, those who sleep little and eat low amount of calories/day (Araujo et al., 2010; Fex et al., 2012; Hansen et al., 2013; Hossain et al., 2012; Saito et al., 2012; Schlüssel et al., 2008).

The relationship between handgrip strength levels and sociodemographic and lifestyle variables has been extensively recorded in literature (Araujo et al., 2010; Hansen et al., 2013; Hossain et al., 2012; Saito et al., 2012; Schlüssel et al., 2008). However, such information is not compiled in systematic reviews or in documents from health authorities that can serve as a guide for health professionals. In this sense, the performance of systematic reviews to gather evidence for these associations is justified for synthesizing in a systematic, objective and conclusive way the association between handgrip strength levels and sociodemographic and lifestyle factors.

The aim of this systematic review was to assess the association between handgrip strength levels and sociodemographic (gender, age, economic status and education level) and lifestyle variables (physical activity, smoking, sleep and food consumption) in the adult population.

\section{Methods}

\section{Search strategy}

The search for studies occurred during the period from January to September 2015 in PubMed, Web of Science, Lilacs, EBSCO, Scopus and Scielo databases.

Pubmed data platform was also searched because it includes about 21 million citations of articles and journals, but the largest database searched was Medline, which indexes 5000 journals published in more than 80 countries (Wheeler et al., 2005); the Web of Science database was used for providing access to more than 9200 titles of journals and 148,000 conference proceedings (Reuters, 2010). The system Bireme, using Lilacs database, was also used for covering more than 350,000 articles from 670 renowned journals in the health area (Health and Services, 1991). For being the world's main database aggregator and offering a suite of over 200 research databases in full text and abstracts, the EBSCO database was also used (Vaughan, 2011). Developed by Elsevier, Scopus database was also searched as it combines the features of PubMed and Web of Science databases, allowing the search of medical literature and academic needs, being the world's largest reference source of technical and scientific literature reviewed by peers (Falagas et al., 2008). Finally, Scielo database provides broad access to scientific journals as a whole (Packer et al., 1998).

Advanced search was used (tool available in databases to perform specific searches using "keywords") from the construction of blocks performed by the researcher. The first block was composed of terms related to handgrip strength (outcome) and the words used with their translations into English and Spanish were: muscle strength; hand strength; hand grip; grip strength; handgrip strength; muscle strength; hand strength; grip strength; handgrip strength, palm grip strength, hand muscle strength dynamometer; manual dynamometer and hand strength dynamometer. The second block was composed of descriptors related to the age of the population being investigated: adult; adults; young adult; young adults. The third block was composed of descriptors related to exposure (sociodemographic factors - man, men, woman, women, age, gender, age groups, age factors, sociodemographic factors, sociodemographic characteristics, sociodemographic data, social factors, socioeconomic factors, educational level, paternal education; maternal education, schooling; paternal schooling; maternal schooling; economy class, economic status, social class, and lifestyle - motor activity, motor activities, physical activity, physical activities, exercise, sleep, sleep duration, short sleep, smoking, tobacco smoking, diet pattern, food intake, eating behavior, eating habits). In order to relate and using at least one word from each block, the term "OR" was used. The term "AND" was used for the purpose of adding at least one word from each block.

For the management of studies found, the EndNote ${ }^{\circledR}$ software was used, whose functions allow the inclusion of 
specific libraries, enabling the division and organization of results from each database.

\section{Inclusion criteria}

Inclusion criteria were: original scientific papers (reviews were excluded) assessing the association between handgrip strength and some of the variables related to sociodemographic factors (age, sex, educational level and economic status) and lifestyle (activity physical, tobacco use, sleep and food intake); cross-sectional studies; studies whose population aged 20-59 years and studies written in English, Portuguese or Spanish. In addition, studies should describe how variables were measured and have used manual dynamometer as tool to evaluate handgrip strength levels. Fig. 1 shows the process of selection and inclusion of studies in this review. All studies that met the inclusion criteria were analyzed fully and independently by two researchers. Each researcher elaborated a table summarizing the information extracted from studies and then data were compared in order to verify the agreement between peers. If there was disagreement, a third evaluator would issue his opinion.

\section{Data extraction}

The main characteristics of studies were extracted and entered into a table (Table 1) with information regarding the author's name (s), place and year of the survey, sample size, average age or age range of participants, data collection instrument, protocol used and average handgrip strength levels, cutoff point used in the classification of handgrip strength levels and sociodemographic and lifestyle variables correlated with the outcome.

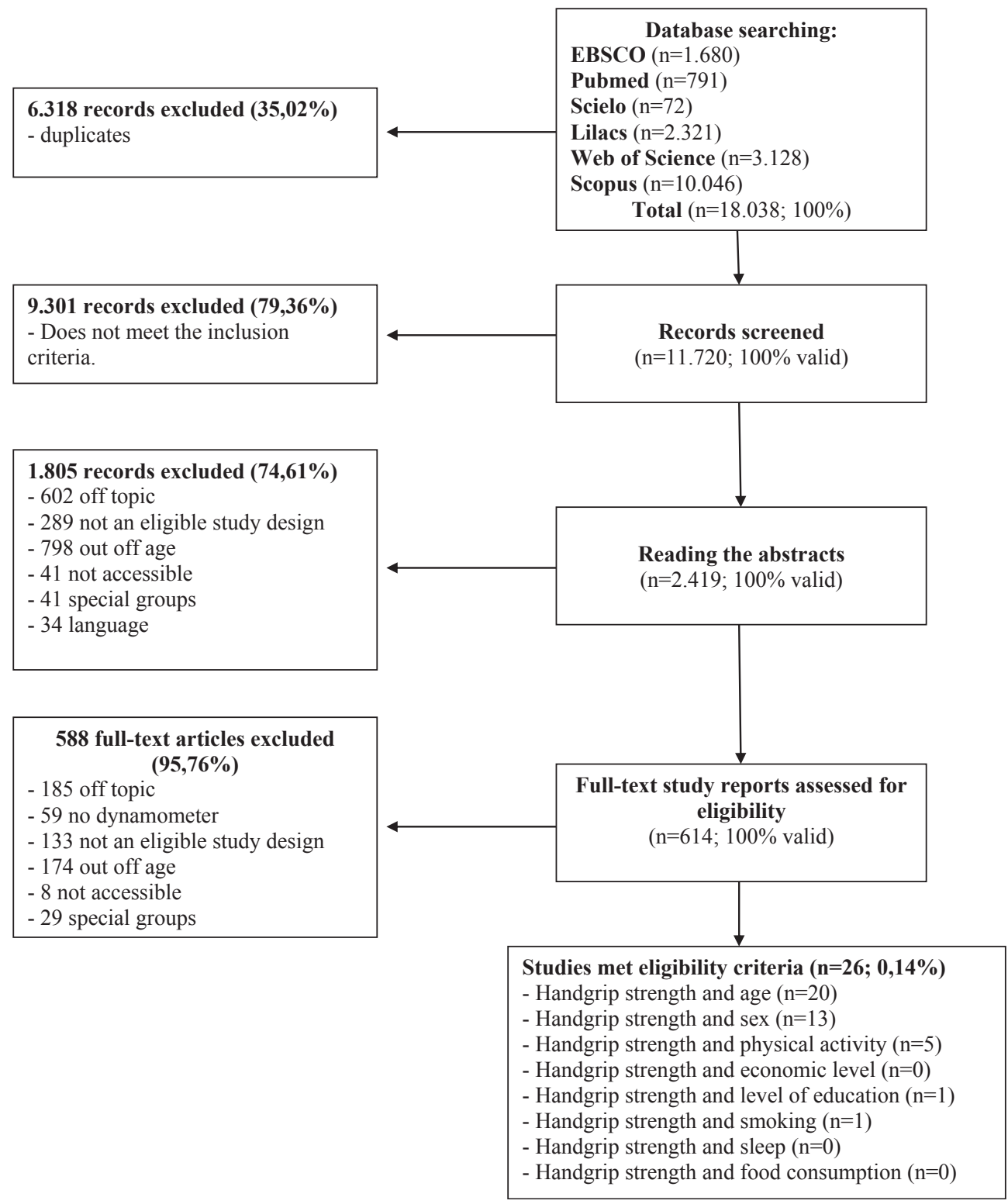

Figure 1 Results of data searches and criteria used to select studies for the review of handgrip strength and sociodemographic and lifestyle. 
Table 1 Studies examining the association between level of muscle strength and sociodemographic factors and lifestyle.

\begin{tabular}{|c|c|c|c|c|c|c|c|}
\hline Reference & $\begin{array}{l}\text { Local area } \\
\text { (study year) }\end{array}$ & $\begin{array}{l}\text { Participants } \\
\text { (age at } \\
\text { baseline: Mean } \\
\text { or range-years) }\end{array}$ & $\begin{array}{l}\text { Measurement of } \\
\text { HGS and } \\
\text { evaluation } \\
\text { position }\end{array}$ & $\begin{array}{l}\text { Trials/hand } \\
\text { used/Scores } \\
\text { used/rest } \\
\text { between each } \\
\text { trial }\end{array}$ & $\begin{array}{l}\text { Mean (kgf or } \\
\text { pounds)/standard } \\
\text { deviation or } \\
\text { standard error }\end{array}$ & $\begin{array}{l}\text { Cut-off } \\
\text { value }\end{array}$ & Outcomes \\
\hline $\begin{array}{c}\text { (Mathiowetz } \\
\text { et al., } \\
\text { 1985) }\end{array}$ & $\begin{array}{l}\text { Milwaukee- } \\
\text { USA }\end{array}$ & $\begin{array}{l}628(20-94 \\
\text { years })\end{array}$ & $\begin{array}{l}\text { Jamar Hand } \\
\text { Dynamometer } \\
\text { Seated and elbow } \\
\text { flexed at } 90^{\circ}\end{array}$ & $\begin{array}{l}\text { Three trials } \\
\text { Highest value }\end{array}$ & 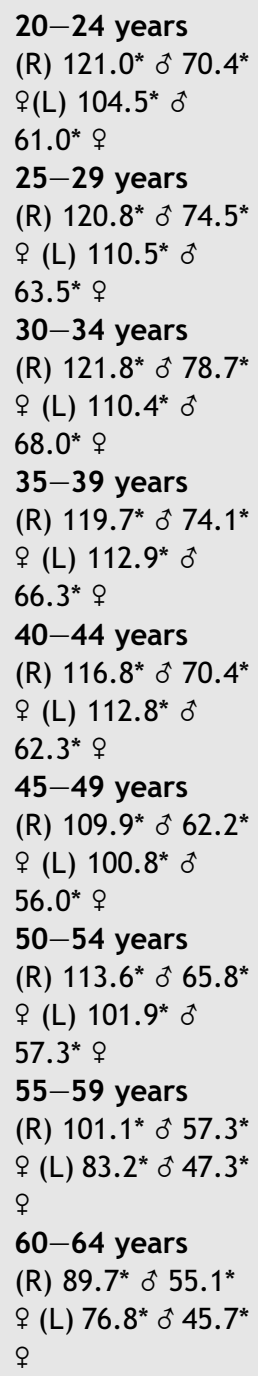 & 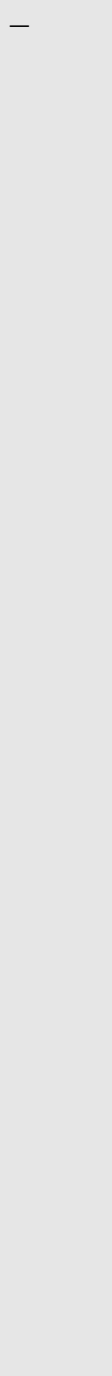 & $\begin{array}{l}\text { - Sex } \\
\text { - Age }\end{array}$ \\
\hline $\begin{array}{l}\text { (Rantanen } \\
\text { et al., } \\
\text { 1992) }\end{array}$ & $\begin{array}{l}\text { Helsinki- } \\
\text { Finland }\end{array}$ & $\begin{array}{l}112 \text { women }(50 \\
-60 \text { years) }\end{array}$ & $\begin{array}{l}\text { Unknown } \\
\text { Seated and } \\
\text { elbow flexed }\end{array}$ & $\begin{array}{l}\text { Three trials } \\
\text { Highest value }\end{array}$ & $\begin{array}{l}\text { Physically } \\
\text { Active/Higher } \\
\text { Education: } \\
385^{*}(78) \\
\text { Physically } \\
\text { Active/Lower } \\
\text { Level of } \\
\text { Education: } \\
367^{*}(74) \\
\text { Sedentary/ } \\
\text { Higher } \\
\text { Education: } \\
343^{*}(86) \\
\text { Sedentary/ } \\
\text { Lower Level of } \\
\text { Education: } \\
329^{*}(89)\end{array}$ & - & $\begin{array}{l}\text { - Physical } \\
\text { Activity } \\
\text { - } \\
\text { Education } \\
\text { Degree }\end{array}$ \\
\hline
\end{tabular}


Table 1 (continued)

\begin{tabular}{|c|c|c|c|c|c|c|}
\hline Reference & $\begin{array}{l}\text { Local area } \\
\text { (study year) }\end{array}$ & $\begin{array}{l}\text { Participants } \\
\text { (age at } \\
\text { baseline: Mean } \\
\text { or range-years) }\end{array}$ & $\begin{array}{l}\text { Measurement of } \\
\text { HGS and } \\
\text { evaluation } \\
\text { position }\end{array}$ & $\begin{array}{l}\text { Trials/hand } \\
\text { used/Scores } \\
\text { used/rest } \\
\text { between each } \\
\text { trial }\end{array}$ & $\begin{array}{l}\text { Mean (kgf or Cut-off } \\
\text { pounds)/standard value } \\
\text { deviation or } \\
\text { standard error }\end{array}$ & Outcomes \\
\hline
\end{tabular}

\begin{tabular}{|c|c|c|c|c|}
\hline $\begin{array}{c}\text { (Chau et al., } \\
\text { 1997) }\end{array}$ & France & $\begin{array}{l}101(38.5 \text { o; } \\
35.5 \text { ㅇ) }\end{array}$ & $\begin{array}{l}\text { Jamar and Collins } \\
\text { Hand } \\
\text { Dynamometer } \\
\text { Standing with the } \\
\text { arm extended and } \\
\text { relaxed alongside } \\
\text { the body }\end{array}$ & $\begin{array}{l}\text { Two trials } \\
\text { Five minutes } \\
\text { rest }\end{array}$ \\
\hline
\end{tabular}

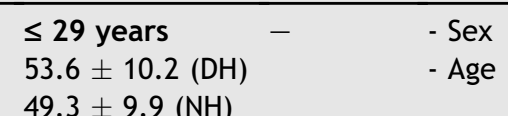

$9.9(\mathrm{NH})$

JAMAR o

$45.5 \pm 9.0(\mathrm{DH})$

$39.4 \pm 9.9(\mathrm{NH})$

COLLINS 0 \%

$34.8 \pm 6.6(\mathrm{DH})$

$32.4 \pm 6.5(\mathrm{NH})$

JAMAR 우

$29.5 \pm 6.8(\mathrm{DH})$

$24.8 \pm 6.5(\mathrm{NH})$

COLLINS 우

30-39 years

$55.5 \pm 8.7(\mathrm{DH})$

$52.9 \pm 9.1(\mathrm{NH})$

JAMAR o

$48.4 \pm 9.3(\mathrm{DH})$

$44.1 \pm 9.9(\mathrm{NH})$

COLLINS o 0

$38.6 \pm 7.7(\mathrm{DH})$

$35.5 \pm 6.9(\mathrm{NH})$

JAMAR $q$

$34.4 \pm 7.0(\mathrm{DH})$

$29.8 \pm 7.2(\mathrm{NH})$

COLLINS 우

40-49 years

$58.1 \pm 9.9(\mathrm{DH})$

$53.6 \pm 7.7(\mathrm{NH})$

JAMAR o

$50.7 \pm 7.4(\mathrm{DH})$

$46.6 \pm 4.6(\mathrm{NH})$

COLLINS 0

$32.5 \pm 5.8(\mathrm{DH})$

$30.8 \pm 5.8(\mathrm{NH})$

JAMAR +

$28.7 \pm 4.7(\mathrm{DH})$

$25.3 \pm 4.0(\mathrm{NH})$

COLLINS 우

$\geq 50$ years

$54.8 \pm 9.3(\mathrm{DH})$

$50.8 \pm 6.6(\mathrm{NH})$

JAMAR o

$49.8 \pm 7.6(\mathrm{DH})$

$44.4 \pm 5.3(\mathrm{NH})$

COLLINS 0

$35.5 \pm 6.1(\mathrm{DH})$

$32.2 \pm 5.0(\mathrm{NH})$

JAMAR +

$29.0 \pm 7.6(\mathrm{DH})$

$26.4 \pm 7.3(\mathrm{NH})$

COLLINS 9 
Table 1 (continued)

\begin{tabular}{|c|c|c|c|c|c|c|c|}
\hline Reference & $\begin{array}{l}\text { Local area } \\
\text { (study year) }\end{array}$ & $\begin{array}{l}\text { Participants } \\
\text { (age at } \\
\text { baseline: Mean } \\
\text { or range-years) }\end{array}$ & $\begin{array}{l}\text { Measurement of } \\
\text { HGS and } \\
\text { evaluation } \\
\text { position }\end{array}$ & $\begin{array}{l}\text { Trials/hand } \\
\text { used/Scores } \\
\text { used/rest } \\
\text { between each } \\
\text { trial }\end{array}$ & $\begin{array}{l}\text { Mean (kgf or } \\
\text { pounds)/standard } \\
\text { deviation or } \\
\text { standard error }\end{array}$ & $\begin{array}{l}\text { Cut-off } \\
\text { value }\end{array}$ & Outcomes \\
\hline $\begin{array}{l}\text { (Caporrino } \\
\text { et al., } \\
\text { 1999) }\end{array}$ & $\begin{array}{l}\text { São Paulo- } \\
\text { Brazil }\end{array}$ & $\begin{array}{l}800(20-60 \\
\text { years })\end{array}$ & $\begin{array}{l}\text { Jamar Hand } \\
\text { Dynamometer- } \\
\text { Seated and elbow } \\
\text { flexed }\end{array}$ & $\begin{array}{l}\text { Three trials } \\
\text { One minute rest }\end{array}$ & $\begin{array}{l}\text { (R) } 44.2 \lesssim 31.6 \% \\
\text { (L) } 40.5 \circlearrowleft 28.4 \%\end{array}$ & - & $\begin{array}{l}\text { - Sex } \\
\text { - Age }\end{array}$ \\
\hline $\begin{array}{l}\text { (Hunter } \\
\text { et al., } \\
2000)\end{array}$ & $\begin{array}{l}\text { Sydney- } \\
\text { Australia }\end{array}$ & $\begin{array}{l}217 \text { women }(20 \\
-89 \text { years) }\end{array}$ & Unknown & Unknown & Unknown & - & $\begin{array}{l}\text { - Age } \\
\text { - Physical } \\
\text { activity }\end{array}$ \\
\hline $\begin{array}{l}\text { (Luna- } \\
\text { Heredia } \\
\text { et al., } \\
\text { 2005) }\end{array}$ & $\begin{array}{l}\text { Móstoles-Spain } \\
\text { (2003) }\end{array}$ & $\begin{array}{l}517 \text { (60.00'; } \\
50.09)\end{array}$ & $\begin{array}{l}\text { Baseline and Grip-D } \\
\text { Hand } \\
\text { Dynamometer }\end{array}$ & Unknown & $\begin{array}{l}22.8 \pm 12.49 \\
35.1 \pm 7.2 \sigma^{\pi}\end{array}$ & - & $\begin{array}{l}\text { - Sex } \\
\text { - Age }\end{array}$ \\
\hline $\begin{array}{l}\text { (Anakwe } \\
\text { et al., } \\
\text { 2007) }\end{array}$ & $\begin{array}{l}\text { Edinburgh- } \\
\text { United } \\
\text { Kingdom. }\end{array}$ & $\begin{array}{l}250 \text { (46.70ే; } \\
39.0 \text { ㅇ) }\end{array}$ & $\begin{array}{l}\text { Jamar Hand } \\
\text { Dynamometer - } \\
\text { Seated }\end{array}$ & Five trials & $\begin{array}{l}48.6 \pm 11.0 \sigma^{\star} \\
28.5 \pm 4.69\end{array}$ & - & - Sex \\
\hline $\begin{array}{l}\text { (Schlussel } \\
\text { et al., } \\
\text { 2008) }\end{array}$ & $\begin{array}{l}\text { Niteroi-Brazil } \\
\text { (2003) }\end{array}$ & 3.050 & $\begin{array}{l}\text { Jamar Hand } \\
\text { Dynamometer - } \\
\text { Standing with the } \\
\text { arm extended and } \\
\text { relaxed alongside } \\
\text { the body }\end{array}$ & One minute rest & $\begin{array}{l}\text { (R) } 42.8 / 0.3 \mathrm{SE} \\
\delta 25.3 / 0.3 \mathrm{SE} q \\
\text { (L) } 40.9 / 0.3 \mathrm{SE} \\
\delta 24.0 / 0.3 \mathrm{SE} q\end{array}$ & - & - Age \\
\hline $\begin{array}{l}\text { (Adedoyin } \\
\text { et al., } \\
\text { 2009) }\end{array}$ & Ile-Ife-Nigeria & $\begin{array}{l}745(20-70 \\
\text { years })\end{array}$ & $\begin{array}{l}\text { Takey Hand } \\
\text { Dynamometer } \\
\text { Seated and elbow } \\
\text { flexed at } 90^{\circ}\end{array}$ & $\begin{array}{l}\text { Three trials } \\
\text { Both Hands- } \\
\text { Mean of scores }\end{array}$ & 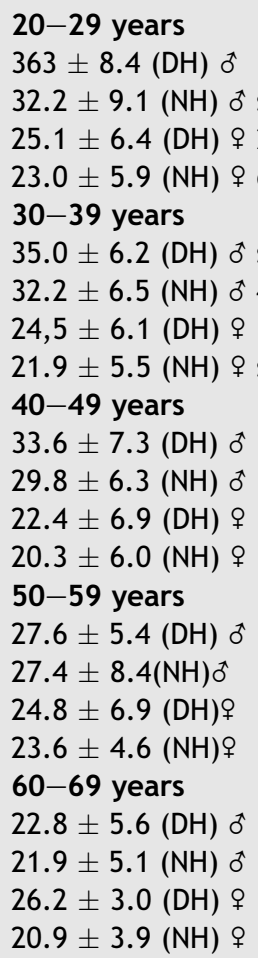 & $\begin{array}{l}1^{0} \text { quartile } \\
\text { (low } \\
\text { strength) } \\
2^{0} / 3^{0} \\
\text { quartiles } \\
\text { (moderate } \\
\text { strength) } \\
4^{0} \text { quartile } \\
\text { (good } \\
\text { strength) }\end{array}$ & $\begin{array}{l}\text { - Sex } \\
\text { - Age }\end{array}$ \\
\hline $\begin{array}{l}\text { (Kaur, } \\
\text { 2009) }\end{array}$ & Haryana-Índia & $\begin{array}{l}600 \text { women }(40 \\
-70 \text { years) }\end{array}$ & Unknown & $\begin{array}{l}\text { Weiner, J.S., } \\
\text { Lourie, J.A., } \\
1981\end{array}$ & $\begin{array}{l}20.3 \pm 5.2 \text { (rural) } \\
18.9 \pm 4.8 \\
\text { (urban) }\end{array}$ & - & - Age \\
\hline $\begin{array}{l}\text { (Werle } \\
\text { et al., } \\
\text { 2009) }\end{array}$ & Switzerland & $\begin{array}{l}1.023(18-96 \\
\text { years) }\end{array}$ & $\begin{array}{l}\text { Jamar Hand } \\
\text { Dynamometer } \\
\text { Seated and elbow } \\
\text { flexed at } 90^{\circ}\end{array}$ & $\begin{array}{l}\text { Three trials- } \\
\text { Mean }\end{array}$ & 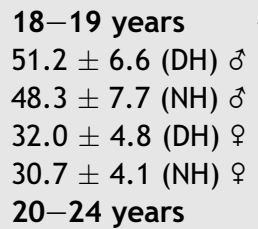 & - & $\begin{array}{l}\text { - Age } \\
\text { - Sex }\end{array}$ \\
\hline
\end{tabular}


Table 1 (continued)

\begin{tabular}{|c|c|c|c|c|c|c|}
\hline Reference & $\begin{array}{l}\text { Local area } \\
\text { (study year) }\end{array}$ & $\begin{array}{l}\text { Participants } \\
\text { (age at } \\
\text { baseline: Mean } \\
\text { or range-years) }\end{array}$ & $\begin{array}{l}\text { Measurement of } \\
\text { HGS and } \\
\text { evaluation } \\
\text { position }\end{array}$ & $\begin{array}{l}\text { Trials/hand } \\
\text { used/Scores } \\
\text { used/rest } \\
\text { between each } \\
\text { trial }\end{array}$ & $\begin{array}{l}\text { Mean (kgf or Cut-off } \\
\text { pounds)/standard value } \\
\text { deviation or } \\
\text { standard error }\end{array}$ & Outcomes \\
\hline
\end{tabular}

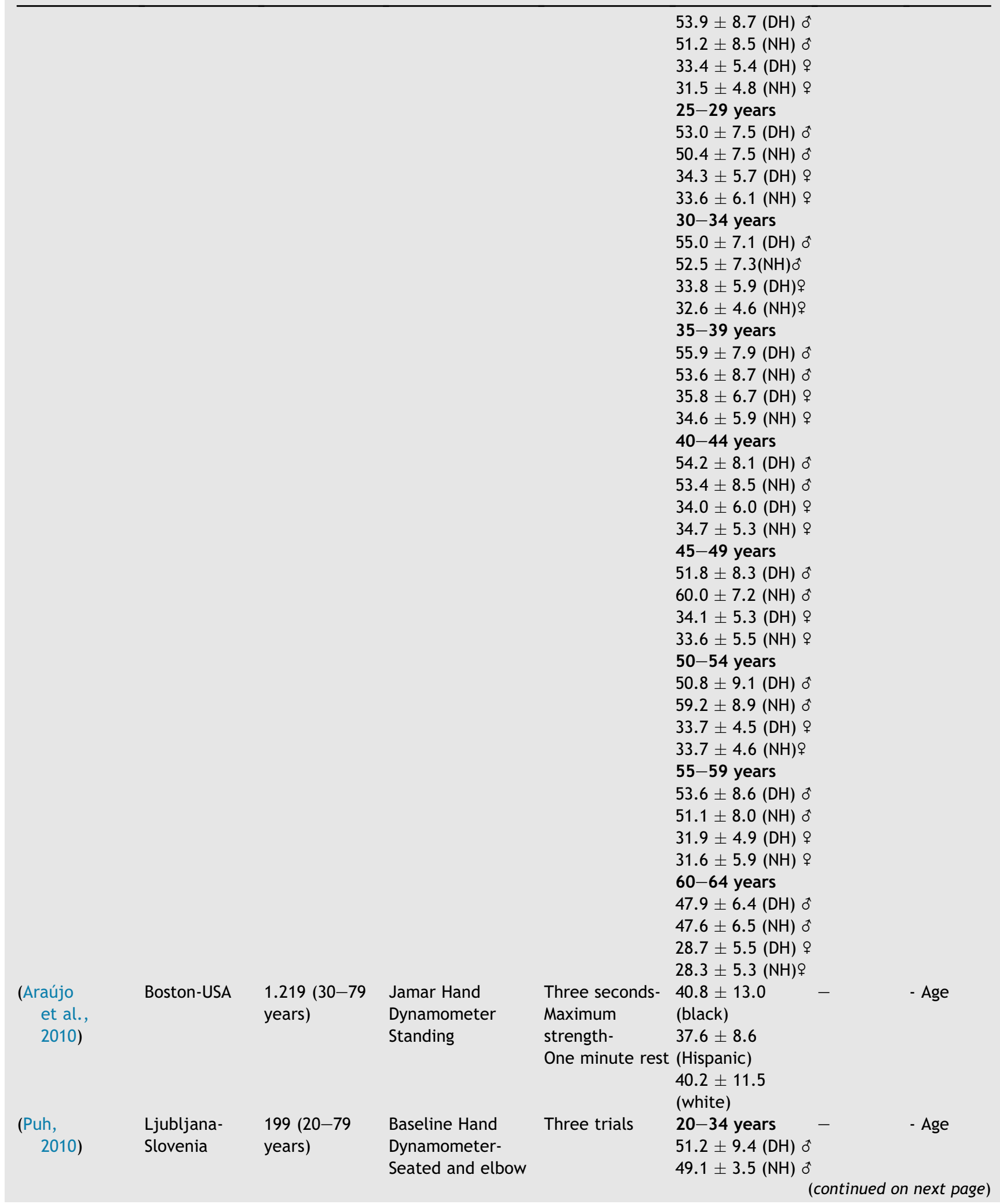


Table 1 (continued)

\begin{tabular}{|c|c|c|c|c|c|c|c|}
\hline Reference & $\begin{array}{l}\text { Local area } \\
\text { (study year) }\end{array}$ & $\begin{array}{l}\text { Participants } \\
\text { (age at } \\
\text { baseline: Mean } \\
\text { or range-years) }\end{array}$ & $\begin{array}{l}\text { Measurement of } \\
\text { HGS and } \\
\text { evaluation } \\
\text { position }\end{array}$ & $\begin{array}{l}\text { Trials/hand } \\
\text { used/Scores } \\
\text { used/rest } \\
\text { between each } \\
\text { trial }\end{array}$ & $\begin{array}{l}\text { Mean (kgf or } \\
\text { pounds)/standard } \\
\text { deviation or } \\
\text { standard error }\end{array}$ & $\begin{array}{l}\text { Cut-off } \\
\text { value }\end{array}$ & Outcomes \\
\hline & & & flexed at $90^{\circ}$ & & 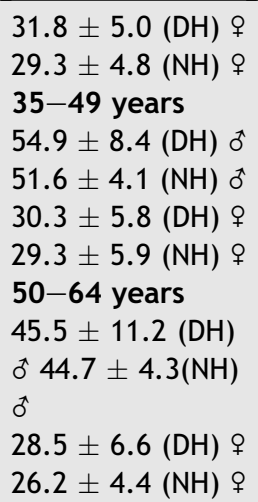 & & \\
\hline $\begin{array}{c}\text { (Aadahl } \\
\text { et al., } \\
\text { 2011) }\end{array}$ & $\begin{array}{l}\text { Copenhagem- } \\
\text { Denmark }\end{array}$ & $\begin{array}{l}3.471 \text { (50.00; } \\
49.09)\end{array}$ & $\begin{array}{l}\text { Jamar Hand } \\
\text { Dynamometer } \\
\text { Seated and elbow } \\
\text { flexed at } 90^{\circ}\end{array}$ & $\begin{array}{l}\text { Three trials/ } \\
\text { Dominant hand } \\
\text { Highest value }\end{array}$ & $\begin{array}{l}49.2 \pm 8.0 \circlearrowleft \\
31.1 \pm 6.19\end{array}$ & - & $\begin{array}{l}\text { - Age } \\
\text { - Physical } \\
\text { Activity }\end{array}$ \\
\hline $\begin{array}{l}\text { (Peters } \\
\text { et al., } \\
\text { 2011) }\end{array}$ & $\begin{array}{l}\text { Maastricht- } \\
\text { Netherlands }\end{array}$ & $\begin{array}{l}720(54.9 \\
\text { years })\end{array}$ & $\begin{array}{l}\text { Jamar Hand } \\
\text { Dynamometer } \\
\text { Seated and elbow } \\
\text { flexed at } 90^{\circ}\end{array}$ & $\begin{array}{l}\text { Three trials } \\
\text { Highest value }\end{array}$ & Unknown & - & - Age \\
\hline $\begin{array}{l}\text { (Hossain } \\
\text { et al., } \\
\text { 2012) }\end{array}$ & $\begin{array}{l}\text { Kuala Lumpur- } \\
\text { Malaysia } \\
(2010)\end{array}$ & $\begin{array}{l}500 \text { (35.16; } \\
34.7 \text { 우) }\end{array}$ & $\begin{array}{l}\text { Jamar Hand } \\
\text { Dynamometer }\end{array}$ & Three trials & $\begin{array}{l}29.8 \pm 8.20 \\
17.6 \pm 5.4 \text { ㅇ }\end{array}$ & Tertile & $\begin{array}{l}\text { - Sex } \\
\text { - Age }\end{array}$ \\
\hline $\begin{array}{l}\text { (Klun et al., } \\
\text { 2012) }\end{array}$ & $\begin{array}{l}\text { Bad } \\
\text { Rappenau- } \\
\text { Germany }\end{array}$ & $\begin{array}{l}750 \text { (40.30; } \\
25.2 \text { 우) }\end{array}$ & $\begin{array}{l}\text { Jamar Hand } \\
\text { Dynamometer } \\
\text { Seated and elbow } \\
\text { flexed at } 90^{\circ}\end{array}$ & Unknown & $\begin{array}{l}43.1 \pm 8.30 \\
25.2 \pm 6.0 \text { o }\end{array}$ & - & $\begin{array}{l}\text { - Sex } \\
\text { - Age }\end{array}$ \\
\hline $\begin{array}{l}\text { (Saito et al., } \\
\text { 2012) }\end{array}$ & $\begin{array}{l}\text { Okayama- } \\
\text { Japan }\end{array}$ & $\begin{array}{l}4.249 \text { men } \\
(43.1 \text { years) }\end{array}$ & $\begin{array}{l}\text { Sakay Hand } \\
\text { Dynamometer }\end{array}$ & $\begin{array}{l}\text { Two trials- } \\
\text { Both hands- } \\
\text { Highest value }\end{array}$ & $\begin{array}{l}\text { NS } 44.9 \pm 8.0(\mathrm{R}) \\
42.9 \pm 7.6(\mathrm{~L}) \\
\text { CS } 43.3 \pm 8.4(\mathrm{R}) \\
41.2 \pm 8.0(\mathrm{~L})\end{array}$ & - & - Smoking \\
\hline $\begin{array}{l}\text { (Zhao et al., } \\
\text { 2012) }\end{array}$ & $\begin{array}{l}\text { Yunnan-China } \\
(2012)\end{array}$ & $\begin{array}{l}109 \text { (43.10; } \\
41.49)\end{array}$ & $\begin{array}{l}\text { Xiangshan Hand } \\
\text { Dynamometer }\end{array}$ & $\begin{array}{l}\text { Three trials- } \\
\text { Both hands- } \\
\text { Highest value }\end{array}$ & $\begin{array}{l}40.2 \pm 7.5 \sigma(\mathrm{R}) \\
24.2 \pm 5.9 \%(\mathrm{R}) \\
38.6 \pm 7.8 \sigma(\mathrm{L}) \\
22.5 \pm 5.8 \text { (L) }\end{array}$ & - & $\begin{array}{l}\text { - Age } \\
\text { - Sex }\end{array}$ \\
\hline $\begin{array}{c}\text { (Hansen } \\
\text { et al., } \\
\text { 2013) }\end{array}$ & $\begin{array}{l}\text { Copenhagen- } \\
\text { Denmark- } \\
(2007)\end{array}$ & $\begin{array}{l}16.539(51.9 \\
\text { years) }\end{array}$ & $\begin{array}{l}\text { Takei Hand } \\
\text { Dynamometer - } \\
\text { Standing with the } \\
\text { arm extended }\end{array}$ & $\begin{array}{l}\text { Two trials- } \\
\text { Both hands- } \\
\text { Highest value }\end{array}$ & 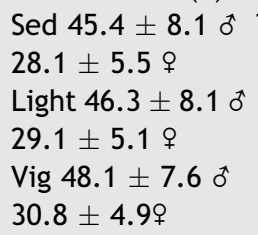 & Tertile & $\begin{array}{l}\text { - Age } \\
\text { - Physical } \\
\text { activity }\end{array}$ \\
\hline $\begin{array}{c}\text { (Montalcini } \\
\text { et al., } \\
\text { 2013) }\end{array}$ & $\begin{array}{l}\text { Catanzaro- } \\
\text { Italy (2011) }\end{array}$ & $\begin{array}{l}335\left(21.40^{7} ;\right. \\
21.4 \text { 우 }\end{array}$ & $\begin{array}{l}\text { Saehan Hand } \\
\text { Dynamometer- } \\
\text { Seated with the } \\
\text { arm extended }\end{array}$ & Highest value & $\begin{array}{l}44.7 \pm 6.60 \\
27.7 \pm 4.3 \subsetneq\end{array}$ & - & - Sex \\
\hline $\begin{array}{l}\text { (Von Hurst } \\
\text { et al., } \\
\text { 2013) }\end{array}$ & $\begin{array}{l}\text { Auckland-New } \\
\text { Zeland }\end{array}$ & $\begin{array}{l}137 \text { women } \\
\text { ( } 23.8 \text { years) }\end{array}$ & $\begin{array}{l}\text { Smedlay Hand } \\
\text { Dynamometer- } \\
\text { Standing with the } \\
\text { elbow flexed at } 90^{\circ}\end{array}$ & $\begin{array}{l}\text { Three trials- } \\
\text { Both hands- } \\
\text { Highest value }\end{array}$ & $\begin{array}{l}27.3 \pm 5.8(\mathrm{DH}) \\
25.6 \pm 5.7(\mathrm{NH})\end{array}$ & - & $\begin{array}{l}\text { - Physical } \\
\text { activity }\end{array}$ \\
\hline $\begin{array}{l}\text { (Zhao et al., } \\
\text { 2013) }\end{array}$ & $\begin{array}{l}\text { Yunnan-China } \\
(2012)\end{array}$ & $\begin{array}{l}140 \text { (37.80; } \\
40.39)\end{array}$ & $\begin{array}{l}\text { Xiangshan Hand } \\
\text { Dynamometer }\end{array}$ & $\begin{array}{l}\text { Three trials- } \\
\text { Both hands- } \\
\text { Highest value }\end{array}$ & 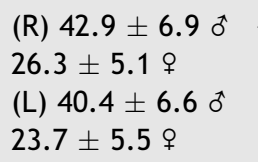 & - & $\begin{array}{l}\text { - Sex } \\
\text { - Age }\end{array}$ \\
\hline
\end{tabular}




\begin{tabular}{|c|c|c|c|c|c|c|c|}
\hline Reference & $\begin{array}{l}\text { Local area } \\
\text { (study year) }\end{array}$ & $\begin{array}{l}\text { Participants } \\
\text { (age at } \\
\text { baseline: Mean } \\
\text { or range-years) }\end{array}$ & $\begin{array}{l}\text { Measurement of } \\
\text { HGS and } \\
\text { evaluation } \\
\text { position }\end{array}$ & $\begin{array}{l}\text { Trials/hand } \\
\text { used/Scores } \\
\text { used/rest } \\
\text { between each } \\
\text { trial }\end{array}$ & $\begin{array}{l}\text { Mean (kgf or } \\
\text { pounds)/standard } \\
\text { deviation or } \\
\text { standard error }\end{array}$ & $\begin{array}{l}\text { Cut-off } \\
\text { value }\end{array}$ & Outcomes \\
\hline $\begin{array}{c}\text { (Abe et al., } \\
\text { 2014) }\end{array}$ & Chiba-Japan & $\begin{array}{l}55 \text { men }(33.3 \\
\text { years) }\end{array}$ & $\begin{array}{l}\text { Takei Hand } \\
\text { Dynamometer- } \\
\text { Standing with the } \\
\text { arm extended. }\end{array}$ & $\begin{array}{l}\text { Two trials } \\
\text { Both hands - } \\
\text { Mean }\end{array}$ & $\begin{array}{l}46 \pm 6 \text { young } \\
43 \pm 6 \text { middle- } \\
\text { age } \\
36 \pm 6 \text { elderly }\end{array}$ & - & - Age \\
\hline (Liao, 2014) & $\begin{array}{l}\text { Tainan-Taiwan } \\
\text { (2014) }\end{array}$ & 200 (20 years) & $\begin{array}{l}\text { Takei Hand } \\
\text { Dynamometer- } \\
\text { Standing with the } \\
\text { arm extended and } \\
\text { relaxed alongside } \\
\text { the body }\end{array}$ & $\begin{array}{l}\text { Five trials } \\
\text { Both Hands- } \\
\text { Highest value }\end{array}$ & 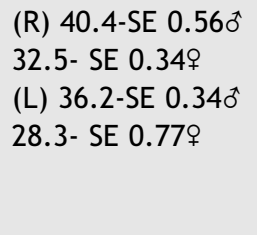 & - & - Sex \\
\hline $\begin{array}{l}\text { (Mohammadian } \\
\text { et al., } \\
\text { 2014) }\end{array}$ & 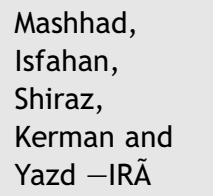 & 1008 (42 years) & $\begin{array}{l}\text { Jamar Hand } \\
\text { Dynamometer } \\
\text { Seated and elbow } \\
\text { flexed at } 90^{\circ}\end{array}$ & $\begin{array}{l}\text { Three trials- } \\
\text { Both hands- } \\
\text { Mean }\end{array}$ & $\begin{array}{l}\text { (DH) } 44.1 \pm 10.8 \\
\delta 26.5 \pm 6.19 \\
\left.(\mathrm{NH}) 42.2 \pm 9.9{ }^{\star}\right) \\
24.6 \pm 5.6 \uparrow\end{array}$ & - & - Age \\
\hline
\end{tabular}

o Man; + Women; Kgf- Kilogram force; * Pounds; (R)- Right Hand; (L)- Left Hand; (DH)- Dominant Hand; (NH)- Non Dominant Hand; (SE)Standard Error; Sed-Sedentary; Vig-Vigorous; NS-Nonsmokers; CS-Current smokers.

\section{Analysis of articles}

To check the consistency of results, the evidence summary for each result was prepared. This summary enables identifying the correlation of findings and is used in reviews in physical activity and health areas (Sallis et al., 2000). The ratio between the number of studies showing some association by the total number of studies found within a given variable was estimated. Based on these studies, each independent variable was classified as: no association $(0-33 \%)$, indeterminate or inconsistent association (34-59\%) and positive or negative consistent association $(\geq 60 \%$ ). The symbol + or - is used to indicate that there was positive or negative association, respectively, in relation to factors investigated, but this association is still not conclusive due to the low number of studies investigating the topic. When four or more studies supported that there would or would not be association, the coding used was ++ or -- (Sallis et al., 2000).

\section{Results}

Six databases were investigated: Pubmed (791 articles), Scielo (72 articles), Web of Science (3128 articles); Ebsco (1680 articles), Lilacs (2321 items) and Scopus (10,046 articles). After inclusion/exclusion, the search resulted in 26 articles (Fig. 1). No studies that met the inclusion criteria of this review found association between handgrip strength levels and economic level, sleep and food intake.

Regarding the place of conduction of these studies, it was found that European countries, with eleven publications and Asian countries, with eight publications, were those that most studied this topic, followed by South American, Oceania, North American and African countries (Table 1).
The association between muscle strength and age was investigated in $76.9 \%$ of studies $(n=20)$ (Tables 1 and 2$)$. All twenty studies demonstrated that increasing age resulted in decreased muscle strength levels (Tables 1 and 2).

The association between muscle strength levels and sex (male/female) was investigated in $50 \%$ of studies $(n=13)$ (Tables 1 and 2). In all surveys, men had higher muscle strength levels compared to women (Tables 1 and 2).

The association between muscle strength levels and physical activity was investigated in five studies (Tables 1 and 2). Physical activity of mild to moderate and moderate to vigorous intensities was associated with higher muscle strength levels (Tables 1 and 2).

Women with higher educational level had higher handgrip strength scores compared to those less educated (Tables 1 and 2). Individuals who smoked cigarettes had lower handgrip strength levels compared to those who did not smoke (Tables 1 and 2). The fact that only one study investigated the association between these variables shows the need for more evidence to confirm this association (Tables 1 and 2).

Several tools to assess strength levels were observed in this review. In twelve studies, $\operatorname{Jamar}^{\circledR}$ dynamometer was used, in four surveys, Takey ${ }^{\circledR}$ dynamometer was used, Baseline $^{\circledR}$ dynamometer was used in two studies, Xiangsham ${ }^{\circledR}$ dynamometer was used in two studies, and Smedlay ${ }^{\circledR}$, Saehan ${ }^{\circledR}$, Sakay ${ }^{\circledR}$ and grip- $D^{\circledR}$ dynamometers were used in one study each (Table 1). In one study, two models of dynamometer were used to measure muscle strength $\left(\right.$ Jamar $^{\circledR}$ and Collins ${ }^{\circledR}$ ) (Table 1). Three studies did not specify the dynamometer brand used (Table 1).

In relation to the position adopted to measure handgrip strength, four different standardizations were verified (Table 1). In ten studies, the subject's position was seated with flexed elbow, four studies used the standing position 
Table 2 Summary of an association between muscular strength levels of handgrip and sociodemographic factors and lifestyle.

\begin{tabular}{|c|c|c|c|c|c|}
\hline Determinant variable & Association positive & Association negative & No relation & $\%$ Studies & Summary code \\
\hline \multicolumn{6}{|l|}{ Activity Intensity } \\
\hline Age & & $\begin{array}{l}\text { (Aadahl et al., 2011; Abe et al., } \\
\text { 2014; } \\
\text { Adedoyin et al., 2009; Araujo } \\
\text { et al., 2010; } \\
\text { Caporrino et al., 1998; Chau } \\
\text { et al., 1997; } \\
\text { Hansen et al., 2013; Hossain } \\
\text { et al., 2012; } \\
\text { Hunter et al., 2000; Kaur, 2009; } \\
\text { Klum et al., 2012;; Luna- } \\
\text { Heredia et al., 2005; } \\
\text { Mathiowetz et al., 1985; } \\
\text { Mohammadian et al., 2014; } \\
\text { Peters et al., 2011; Puh, 2010; } \\
\text { Schlüssel et al., 2008; } \\
\text { Werle et al., 2009; Zhao et al., } \\
\text { 2012; } \\
\text { Zhao et al., 2013) }\end{array}$ & & $100 \%$ & -- \\
\hline Sex (Male) & $\begin{array}{l}\text { (Adedoyin et al., 2009; Anakwe } \\
\text { et al., 2007; } \\
\text { Caporrino et al., 1998; Chau } \\
\text { et al., 1997; } \\
\text { Hossain et al., 2012; Klum } \\
\text { et al., 2012; } \\
\text { Liao, } 2014 \text { Luna-Heredia et al., } \\
\text { 2005; Mathiowetz et al., 1985; } \\
\text { Montalcini et al., 2013; Werle } \\
\text { et al., 2009; } \\
\text { Zhao et al., 2012; Zhao et al., } \\
\text { 2013) }\end{array}$ & & & $100 \%$ & ++ \\
\hline Level of Education & (Rantanen et al., 1992) & & & $100 \%$ & + \\
\hline Economic Level & 0 & 0 & 0 & 0 & * \\
\hline Physical Activity & $\begin{array}{l}\text { (Rantanen et al., 1992) } \\
\text { Unknown } \\
\text { (Hunter et al., 2000b) } \\
\text { Unknown } \\
\text { (Aadahl et al., 2011) } \\
\text { Unknown. } \\
\text { (Hansen et al., 2013a) } \\
\text { LM/MV } \\
\text { (von Hurst et al., 2013) } \\
\text { LM }\end{array}$ & & & $100 \%$ & ++ \\
\hline Smoking & & (Saito et al., 2012) & & $100 \%$ & - \\
\hline Sleep & 0 & 0 & 0 & 0 & * \\
\hline Food Consumption & 0 & 0 & 0 & 0 & * \\
\hline
\end{tabular}

+ Positive association $(60 \%-100 \%)$; - Negative association $(60 \%-100 \%) ;++$ or -- ; When four or more studies supported an association or no association; 0 No association; LM- Light to moderate; MV- Moderate to vigorous.

with arm outstretched, two studies used the sitting position with arm outstretched and the standing position with flexed elbow was adopted in one study (Table 1). In nine studies, the description of the evaluation standard in relation to the subject's position was not verified or specified in detail (Table 1).
Among the studies included in this review, the use of cutoff point for the classification of studies in relation to the strength scores was not verified. Most of them used central tendency measures (mean and median) to present the results obtained $(n=22)$ (Table 1$)$. In two studies, tertiles were used to define subjects classified according to 
strength levels (Table 1). In one study, quartiles were used to classify strength levels (Table 1 ).

\section{Discussion}

The main findings were that the higher the age, the lower the muscle strength levels; men showed greater handgrip strength scores compared to women; physically active individuals had higher handgrip strength levels when compared to those insufficiently active.

The plausibility of the association between lower strength levels with advancing age is related to changes in neuromuscular and endocrine system that occurs with advancing age. The decrease of fast-twitch fibers is among these changes, reducing the total number of muscle fibers, declining the activation of agonist muscles and increasing the request of antagonist muscles and lower activation capacity of motor units (Gale et al., 2007).

Men have more muscle mass than women and this can be a justification for the evidence reported in this review (Valentine et al., 2009). These differences are attenuated when comparisons between sexes are conducted in accordance with values related to body mass (Valentine et al., 2009). Another factor directly associated with higher strength levels in men would be the higher plasma concentrations of the major anabolic hormones (testosterone, GH and IGF-1) in men (Montalcini et al., 2012).

Regular physical activity (mild to moderate or moderate to vigorous) was associated with higher muscle strength levels. The stimuli from physical activity are directly related to the response of the skeletal muscle and adjustments from these stimuli, resulting in increased muscle mass and higher strength levels (Adedoyin et al., 2009). In addition, this review found a study in which subjects who performed physical activity at a higher intensity had higher strength levels (Hansen et al., 2013).

Higher schooling contributed to greater handgrip strength levels. Smoking was related to lower muscle and handgrip strength levels. However, these results should be analyzed with caution, since only one study, for each of these variables, examined such relationships. Further studies are required to investigate the conclusive relationships among these variables.

Regarding the place of conduction of studies included in this review, it was found that European and Asian countries accounted for approximately $73 \%$ of studies. Muscle strength has been used as a diagnostic tool for health problems; thus, the high number of studies in these regions addressing such associations could be justified by the importance given by these countries to health indicators, such as handgrip strength (Sadovsky et al., 2015).

The various standards adopted to classify handgrip strength scores described in the studies included in this review make the comparison of results difficult and indicate lack of consensus on the best standardization to be use. Studies determining specific cutoff points based on a benchmark related to the study population would be required.

In this review, there was variation in the use of different dynamometer brands. This variation is also a limitation when comparing results. The dynamometer should have four important properties: to be able to replicate and determine grip strength with accuracy; to be independent of the hand size; to be comfortable to use and to be small enough to be used in field trials (Caporrino et al., 1998). In this sense, the Jamar $^{\circledR}$ dynamometer was considered the "gold standard" for validation studies compared to other tools to evaluate hand strength, since according to literature, for presenting sealed hydraulic system and no mechanical failures, it provides more accurate measurements (España-Romero et al., 2010; Mathiowetz et al., 1985).

The adoption of various standardization in relation to the subject's position to run the test was a result of this review. This discrepancy in standardization can influence the results observed, since literature has shown higher handgrip strength scores in individuals in the standing position, compared with those in the sitting position (Balogun et al., 1991), in which the justification for these findings would be that the activation of the alpha and gamma motor system, responsible for the strong contraction of extrafusal muscle fibers would be enhanced by the synergistic effect of the lower limb muscles in the standing position (Balogun et al., 1991). However, the most appropriate position to verify handgrip levels is uncertain (España-Romero et al., 2010), since there is still no consensus in literature.

Among the positive aspects observed in this review, the search strategy of studies in Portuguese, English and Spanish can be highlighted. Another positive point is the fact that to date, no systematic reviews investigating sociodemographic and lifestyle factors related to handgrip strength levels in adults have been found. The use of the evidence summary to measure the association of variables was another positive aspect of this review, since it allows identifying conclusive relationships.

The inclusion of only cross-sectional studies was a study limitation, in which aspects of the population are described in a single moment, which ultimately prevents the establishment of causal inferences. Another limitation of this study was the exclusion of other electronic databases.

\section{Conclusion}

In view of the inconclusive results regarding the direction of the associations between handgrip strength, education level and smoking (only one study sought to show the relationship of these factors with the outcome), as well as failing to identify studies that aimed to investigate the relationship the handgrip scores with other sociodemographic and lifestyle variables (economic level, hours of sleep and food intake), it is suggested further research in regard to the relationship of these variables with handgrip strength scores, since by these indicators it is possible to list the specific subgroups with lower levels of force and act in a direct way in the mechanisms related with low power levels handgrip.

Through systematic gathering of scientific evidence about the association between handgrip strength and sociodemographic and lifestyle factors, this research will serve as a support for decision making and health interventions and will provide information able of being reproduced. Interventions in adults aiming to increase strength levels and prevention of diseases caused by this 
condition should be performed, with special attention to females, elderly and those physically inactive.

\section{Conflict of interest}

The authors declare that there is no conflict of interest.

\section{Acknowledgments}

Not applicable.

\section{References}

Aadahl, M., Beyer, N., Linneberg, A., Thuesen, B.H., Jørgensen, T., 2011. Grip strength and lower limb extension power in 19-72year-old Danish men and women: the Health2006 study. BMJ Open 1, e000192. http://dx.doi.org/10.1136/bmjopen-2011000192.

Abe, T., Ogawa, M., Thiebaud, R.S., Loenneke, J.P., Mitsukawa, N., 2014. Is muscle strength ratio a criterion for diagnosis of sitespecific muscle loss? Geriatrics Gerontol Int. 14, 837-844. http://dx.doi.org/10.1111/ggi.12179.

Adedoyin, R.A., Ogundapo, F.A., Mbada, C.E., Adekanla, B.A., Johnson, O.E., Onigbinde, T.A., Emechete, A.A., 2009. Reference values for handgrip strength among healthy adults in Nigeria. Hong Kong Physiother. J. 27, 21-29. http: //dx.doi.org/10.1016/S1013-7025(10)70005-1.

Anakwe, R., Huntley, J., McEachan, J., 2007. Grip strength and forearm circumference in a healthy population. J. Hand Surg. Eur. Vol 32, 203-209. http://dx.doi.org/10.1016/J.JHSB. 2006.11.003.

Araujo, A.B., Chiu, G.R., Kupelian, V., Hall, S.A., Williams, R.E., Clark, R.V., McKinlay, J.B., 2010. Lean mass, muscle strength, and physical function in a diverse population of men: a population-based cross-sectional study. BMC Public Health 10, 508. http://dx.doi.org/10.1186/1471-2458-10-508.

Balogun, J.A., Akomolafe, C.T., Amusa, L.O., 1991. Grip strength: effects of testing posture and elbow position. Archives Phys. Med. Rehabilitation 72, 280-283.

Caporrino, F.A., Faloppa, F., Santos, J.B.G., Réssio, C., Soares, F.H.C., Nakachima, L.R., Segre, N.G., 1998. Estudo populacional da força de preensäo palmar com dinamômetro Jamar. Rev. Bras. Ortop. 33, 150-154.

Chau, N., Bourgkard, E., Pétry, D., Huguenin, P., Remy, E., Andre, J., 1997. Comparison between estimates of hand volume and hand strengths with sex and age with and without anthropometric data in healthy working people. Eur. J. Epidemiol. 13, 309-316.

España-Romero, V., Ortega, F.B., Vicente-Rodríguez, G., Artero, E.G., Rey, J.P., Ruiz, J.R., 2010. Elbow position affects handgrip strength in adolescents: validity and reliability of Jamar, DynEx, and TKK dynamometers. J. Strength \& Cond. Res. 24, 272-277. http://dx.doi.org/10.1519/JSC.0b013e3181b2 $96 a 5$.

Falagas, M.E., Pitsouni, E.I., Malietzis, G.A., Pappas, G., 2008. Comparison of PubMed, Scopus, web of science, and Google scholar: strengths and weaknesses. FASEB J. 22, 338-342.

Fex, A., Artigas, S.B., Dupontgand, S., Filion, M.E., Karelis, A.D., Leheudre, M.A., 2012. Relationship between long sleep duration and functional capacities in postmenopausal women. J. Clin. Sleep Med. 8, 309-313. http://dx.doi.org/10.5664/jcsm.1922.

Gale, C.R., Martyn, C.N., Cooper, C., Sayer, A.A., 2007. Grip strength, body composition, and mortality. Int. J. Epidemiol. 36, 228-235.
Hansen, A.W., Beyer, N., Flensborg-Madsen, T., Grønbæk, M., Helge, J.W., 2013. Muscle strength and physical activity are associated with self-rated health in an adult Danish population. Prev. Med. 57, 792-798. http://dx.doi.org/10.1016/j.ypmed. 2013.08.029.

Health, UDo, Services, H., 1991. Healthy People 2000: National Health Promotion and Disease Prevention Objectives, Healthy People 2000: National Health Promotion and Disease Prevention Objectives. US Government Printing Office.

Hossain, M.G., Zyroul, R., Pereira, B., Kamarul, T., 2012. Multiple regression analysis of factors influencing dominant hand grip strength in an adult Malaysian population. J. Hand Surg. Eur. Vol 37, 65-70. http://dx.doi.org/10.1177/1753193411414639.

Hunter, S.K., Thompson, M.W., Adams, R.D., 2000. Relationships among age-associated strength changes and physical activity level, limb dominance, and muscle group in women. Journals Gerontology Ser. Biol. Sci. Med. Sci. 55, B264-B273.

Kaur, M., 2009. Age-related changes in hand grip strength among rural and urban Haryanvi Jat females. HOMO-J. Comp. Hum. Biol. 60, 441-450. http://dx.doi.org/10.1016/j.jchb. 2009.06.002.

Klum, M., Wolf, M.B., Hahn, P., Leclère, F.M., Bruckner, T., Unglaub, F., 2012. Predicting grip strength and key pinch using anthropometric data, DASH questionnaire and wrist range of motion. Archives Orthop. Trauma Surg. 132, 1807-1811. http: //dx.doi.org/10.1007/s00402-012-1602-8.

Leong, D.P., Teo, K.K., Rangarajan, S., Lopez-Jaramillo, P., Avezum, A., Orlandini, A., Seron, P., Ahmed, S.H., Rosengren, A., Kelishadi, R., 2015. Prognostic value of grip strength: findings from the Prospective Urban Rural Epidemiology (PURE) study. Lancet 386, 266-273. http: //dx.doi.org/10.1016/s0140-6736(14)62000-6.

Liao, K.H., 2014. Experimental study on gender differences in hands and sequence of force application on grip and hand-grip control. Int. J. Occup. Saf. Ergonomics 20, 77-90.

Luna-Heredia, E., Martín-Peña, G., Ruiz-Galiana, J., 2005. Handgrip dynamometry in healthy adults. Clin. Nutr. 24, 250-258.

Mathiowetz, V., Kashman, N., Volland, G., Weber, K., Dowe, M., Rogers, S., 1985. Grip and pinch strength: normative data for adults. Archives Phys. Med. Rehabilitation 66, 69-74.

Mohammadian, M., Choobineh, A., Haghdoost, A., Hasheminejad, N., 2014. Normative data of grip and pinch strengths in healthy adults of Iranian population. Iran. J. Public Health 43, 1113-1122.

Montalcini, T., Migliaccio, V., Ferro, Y., Gazzaruso, C., Pujia, A., 2012. Androgens for postmenopausal women's health? Endocrine 42, 514-520. http://dx.doi.org/10.1007/s12020-0129692-1.

Montalcini, T., Migliaccio, V., Yvelise, F., Rotundo, S., Mazza, E., Liberato, A., Pujia, A., 2013. Reference values for handgrip strength in young people of both sexes. Endocrine 43, 342-345. http://dx.doi.org/10.1007/s12020-012-9733-9.

Packer, A.L., Biojone, M.R., Antonio, I., Takenaka, R.M., García, A.P., ACd, Silva, Murasaki, R.T., Mylek, C., Reis, O.C., Delbucio, H., 1998. SciELO: uma metodologia para publicação eletrônica. Ciência Informação 27, 109-121.

Peters, M.J., van Nes, S.I., Vanhoutte, E.K., Bakkers, M., van Doorn, P.A., Merkies, I.S., Faber, C.G., 2011. Revised normative values for grip strength with the Jamar dynamometer. J. Peripher. Nerv. Syst. 16, 47-50. http://dx.doi.org/10.1111/ j.1529-8027.2011.00318.x.

Puh, U., 2010. Age-related and sex-related differences in hand and pinch grip strength in adults. Int. J. Rehabilitation Res. 33, 4-11. http://dx.doi.org/10.1097/MRR.0b013e328325a8ba.

Rantanen, T., Parkatti, T., Heikkinen, E., 1992. Muscle strength according to level of physical exercise and educational background in middle-aged women in Finland. Eur. J. Appl. Physiol Occup. Physiol 65, 507-512. 
Reuters, T., 2010. Web of Science 2010.

Sadovsky, A.D.I., Poton, W.L., Reis-Santos, B., Barcelos, M.R.B., Silva, I.C.M., 2015. Human Development Index and secondary prevention of breast and cervical cancer: an ecological study. Cad. Saúde Pública 31, 1539-1550. http://dx.doi.org/10. 1590/0102-311X00073014.

Saito, T., Miyatake, N., Sakano, N., Oda, K., Katayama, A., Nishii, K., Numata, T., 2012. Relationship between cigarette smoking and muscle strength in Japanese men. J. Prev. Med. Public Health 45, 381-386. http://dx.doi.org/10.3961/ jpmph.2012.45.6.381.

Sallis, J.F., Prochaska, J.J., Taylor, W.C., 2000. A review of correlates of physical activity of children and adolescents. Med. Sci. Sports Exerc. 32, 963-975.

Schlüssel, M.M., dos Anjos, L.A., de Vasconcellos, M.T.L., Kac, G., 2008. Reference values of handgrip dynamometry of healthy adults: a population-based study. Clin. Nutr. 27, 601-607. http: //dx.doi.org/10.1016/j.clnu.2008.04.004.

Valentine, R.J., Misic, M.M., Rosengren, K.S., Woods, J.A., Evans, E.M., 2009. Sex impacts the relation between body composition and physical function in older adults. Menopause (New York, NY) 16, 518-523. http://dx.doi.org/10.1097/ gme.0b013e31818c931f.
Vaughan, J., 2011. EBSCO discovery services. Libr. Technol. Rep. 47, 30-38.

Von Hurst, P., Conlon, C., Foskett, A., 2013. Vitamin D status predicts hand-grip strength in young adult women living in Auckland, New Zealand. J. steroid Biochem. Mol. Biol. 136, 330-332. http://dx.doi.org/10.1016/j.jsbmb.2012.11.015.

Werle, S., Goldhahn, J., Drerup, S., Simmen, B., Sprott, H., Herren, D., 2009. Age-and gender-specific normative data of grip and pinch strength in a healthy adult Swiss population. J. Hand Surg. Eur. Vol 0, 1-9. http://dx.doi.org/10.1177/ 1753193408096763.

Wheeler, D.L., Barrett, T., Benson, D.A., Bryant, S.H., Canese, K., Church, D.M., DiCuccio, M., Edgar, R., Federhen, S., Helmberg, W., 2005. Database resources of the national center for biotechnology information. Nucleic Acids Res. 33, 39-45.

Zhao, D., Li, B., Yu, K., Zheng, L., 2012. Digit ratio (2D: 4D) and handgrip strength in subjects of Han ethnicity: impact of sex and age. Am. J. Phys. Anthropol. 149, 266-271. http: //dx.doi.org/10.1002/ajpa.22130.

Zhao, D., Yu, K., Zhang, X., Zheng, L., 2013. Digit ratio (2D: 4D) and handgrip strength in Hani ethnicity. PloS one 8, e77958. http: //dx.doi.org/10.1371/journal.pone.0077958.t001. 